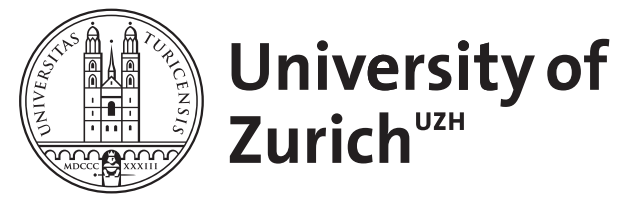

Zurich Open Repository and Archive

University of Zurich

University Library

Strickhofstrasse 39

CH-8057 Zurich

www.zora.uzh.ch

Year: 2013

„Freiheit“ im produktiven Spannungsfeld religiöser und politischer Bildung

Schlag, Thomas

Abstract: Was man von Joachim Gaucks protestantischer Freiheitsgeschichte erfahren und lernen kann

Posted at the Zurich Open Repository and Archive, University of Zurich

ZORA URL: https://doi.org/10.5167/uzh-79537

Journal Article

Published Version

Originally published at:

Schlag, Thomas (2013). „Freiheit“ im produktiven Spannungsfeld religiöser und politischer Bildung. Zeitschrift für Pädagogik und Theologie, 65(2):154-167. 
Gott steht. Der Prozess, durch den der Mensch wirklich frei wird, ist „Bildung“ Bildung verstanden als Transformation von Dogmatik und Ethik in den Lebenslauf, Bildung verstanden als Aufbau einer aus der Kombination von Dogmatik und Ethik gespeisten Urteilskompetenz in innerweltlicher Hinsicht. In diesem Sinne ist Bildung implizit immer von der Frage nach letzten Autoritäten einerseits (objektiv) und eben dem Verhalten zu diesen andererseits (selbstreflexiv) getragen. In anthropologischer Hinsicht führt der pragmatische Atheismus dazu, Freiheit als Geschenk jenseits des eigenen Deutungs- und Leistungsvermögens zu begreifen. Pragmatischer Atheismus ist damit zugleich ein elementarer Ermöglichungsgrund und eine Ausdrucksform religiöser Bildung.

Thomas Schlag

\title{
„Freiheit" im produktiven Spannungsfeld religiöser und politischer Bildung
}

\author{
Was man von Joachim Gaucks protestantischer Freiheitsgeschichte \\ erfahren und lernen kann
}

\section{Einleitung}

Der Zusammenhang von religiöser und politischer Bildung ist innerhalb der religionspädagogischen Fachdebatten in jüngster Zeit wieder deutlicher in das Blickfeld gerückt. Dass religiöse Bildung am Ort der Schule nicht ohne ihre ethischen und politischen Implikationen gedacht werden kann bzw. die Religionsdidaktik wieder stärker auf diese Dimension hin zu profilieren ist, wird von verschiedenen Seiten her und auch in durchaus unterschiedlicher Nuancierung vertreten. ${ }^{1}$ In diesem Zusammenhang ist allerdings bisher kaum eine explizite Auseinandersetzung mit der Freiheitsthematik erfolgt. ${ }^{2}$

\footnotetext{
${ }^{1}$ Vgl. v. a. die in der ZPT dokumentierte Diskussion zwischen Folkert Rickers, Bernhard Grümme und Bernhard Dressler, Politik - Religion, Pädagogik - eine Diskussion. In: ZPT 64 (2012), 279-299 im Anschluss an meine Habilitationsschrift Horizonte demokratischer Bildung. Religionspädagogik in politischer Perspektive. Freiburg/Basel/Wien 2010 sowie B. Grümme, Religionsunterricht und Politik. Bestandsaufnahme - Grundsatzüberlegungen - Perspektiven für eine politische Dimension des Religionsunterrichts. Stuttgart 2009, jüngst Dietrich Zilleßen, Religion und Politik. Wie offen ist der Religionsunterricht? (2012, noch unveröffentlichtes Manuskript) und soeben in dezidierter Ausrichtung G. Lämmermann, Konstruktiv-kritische Religionsdidaktik, in: B. Grümme u.a. (Hg.), Religionsunterricht neu denken. Innovative Ansätze und Perspektiven der Religionsdidaktik. Stuttgart 2012, 29-42.

${ }^{2}$ Eine gewisse Ausnahme mag die soeben erschienene Habilitationsschrift des katholischen Theologen Paul Platzbecker darstellen, in der eine Religionspädagogik in fundamentaltheologisch-hermeneutischer und freiheitstheoretischer Orientierung entfaltet sowie religiöse Bildung als „Freiheitsgeschehen“ entworfen wird; allerdings fehlen Hinweise auf mögliche Zusammenhänge zur Politikdidaktik und politikrelevante Bezüge fast durchgängig, vgl. P. Platzbecker, Religiöse Bildung als Freiheitsgeschehen. Konturen einer religionspädagogischen Grundlagentheorie. Stuttgart 2013.
} 
Eine intensivere Beschäftigung mit Freiheit als anthropologischer und hermeneutischer Grundkategorie vermag nun aber die weitere Fachdebatte und das jeweilige Unterrichtsgeschehen erheblich zu bereichern und ohne diese bleibt eine wesentliche Dimension politisch relevanter religiöser Bildung unberücksichtigt. Denn im Freiheitsthema verbinden sich religiöse und politische Bildung zum einen darin, dass mitmenschliches Denken und Handeln in individuellem und gemeinschaftsorientiertem Sinn befördert werden soll. Zum anderen sind beide fachdidaktischen Zugänge auch im Blick auf die Zielsetzung einer kritischen Bildung hin gleichsam koalitionsfähig: Die jeweiligen Bildungsprozesse sollen dazu verhelfen, dass jene Zustände in kompetenter Weise kritisch benannt, reflektiert und beurteilt werden können, die die freie Entfaltung eigenständigen Denkens und Handelns willkürlich begrenzen, Unfreiheit erzeugen und Mitmenschlichkeit verhindern. ${ }^{3}$

Insofern handelt es sich bei der Freiheitsthematik um eine ebenso zentrale pädagogische Kategorie wie Herausforderung. Dadurch kann der theologische und der allgemeinbildende Charakter ebenso wie das fächerübergreifende Potenzial des Religionsunterrichts als „ein die Freiheit des Heranwachsenden unbedingt bejahendes Handeln“4 bis hin zu seiner Bedeutung für Schulkultur und Schulleben deutlich gemacht werden. Kurz gesagt: Die Grundfrage nach einem politisch relevanten Kompetenzerwerb durch schulische religiöse Bildung lässt sich anhand der Freiheitsthematik in besonders nahe gehender und existenzieller Weise bearbeiten.

Nun stellt sich die Frage, wie eine solche kritische Reflexion und Urteilsbildung so motivierend wie möglich initiiert und konkretisiert werden kann. Denn dass es im unterrichtlichen Bildungsgeschehen mit theoretisch-terminologischen Klärungsversuchen des Freiheitsthemas nicht sein Bewenden haben kann, ist offenkundig.

Deshalb soll in diesem Beitrag der Versuch unternommen werden, im Blick auf die protestantische Berufsbiographie Joachim Gaucks und dessen Überlegungen zum Freiheitsbegriff die religionspädagogische Auseinandersetzung mit diesem Thema am konkreten Beispiel durchzubuchstabieren (2.). Anhand der gegenwärtig so präsenten wie prominenten Figur Joachim Gauck kann der Zusammenhang von religiöser Grundhaltung und politischem Handeln anschaulich nachgezeichnet werden, und so der Zusammenhang zwischen einem evangelisch gegründeten und einem politisch manifesten Freiheitsverständnis auch religionsdidaktisch produktiv gemacht werden (3). Von dort aus sollen religionsdidaktische Folgerungen für die weitergehende Frage des Kompetenzerwerbs im Religionsunterricht skizziert werden (4.). Damit soll in einem weiterreichenden Sinn die Integration des Politischen in religiöse Bildungsprozesse hinein argumentativ plausibel gemacht und verstärkt werden.

Hinter diesem gesamten Vorgehen steht die These, dass kompetenzorientierte Bildungsprozesse durch die Orientierung an einer konkreten Person und deren Biografie

\footnotetext{
${ }^{3}$ Vgl. in politikdidaktischer Hinsicht GPJE (Hg.), Anforderungen an Nationale Bildungsstandards für den Fachunterricht in Politischer Bildung an Schulen. Ein Entwurf. Schwalbach/Ts. ${ }^{2} 2004$.

${ }^{4}$ Norbert Mette, Art. Freiheit, in: N. Mette/F. Rickers (Hg.), Lexikon der Religionspädagogik Bd. 1. Neukirchen-Vluyn 2001, 617.
} 
sowie der aktualitätsbezogenen Kontextualisierung besonders anschaulich sein und auch entsprechende Wirkungen entfalten bzw. einen besonderen Bildungseindruck hinterlassen können. ${ }^{5}$ Es geht folglich darum, was sich für konkrete Bildungsprozesse davon lernen lässt, wie ein „ehemaliger“ Pastor und Repräsentant des ostdeutschen Aufbruchs und nun gegenwärtiger Bundespräsident den Zusammenhang der eigenen christlichen Haltung mit seinem politischen Engagement verknüpft und welche systematischen Begründungen dies hinsichtlich der Freiheitsthematik erfährt.

Von dort her kann erörtert werden, wie sich in dieser protestantischen Biografie und den entsprechenden Äußerungen der spannungsvolle und gleichsam doppelpolige Zusammenhang zwischen christlichem Freiheitsverständnis und einem menschenrechtlich-politischen Verständnis von Freiheit identifizieren lässt. Denn unverkennbar wird Gauck öffentlich nicht nur als charismatischer Grenzgänger, sondern gelegentlich sogar als Repräsentant der Machtkoalition zwischen Kirche und Staat wahrgenommen, was dann die entsprechenden Beschreibungen nach sich zieht: „Wo Joachim Gauck in offizieller Mission auftaucht, teilen sich vor ihm die Massen wie die Wogen einst vor Moses. Gauck schreitet dann huldvoll die Reihen entlang, nickt freundlich lächelnd mal hierhin, mal dorthin und scheint ansonsten ganz ergriffen zu sein, von sich und seiner eigenen Bedeutung. Ich, Gauck, Pfarrer und Präsident“. ${ }^{6}$

Dieser biographiebezogene Zugang zu einer Freiheits-Bildung legt sich aber vor allem deshalb nahe, weil bei Gauck als „Liebhaber der Freiheit“7 eben eine vielfache Bezugnahme auf den Begriff und die Bedeutung der Freiheit als „das Allerwichtigste im Zusammenleben“8 ${ }^{8}$ zu entdecken ist. Im symbolischen Akt seines Eintrags als erstmaliger Kandidat zum Bundespräsidenten in das Gästebuch der Universität Rostock am 12.6.2010 findet sich brennpunktartig das Credo: „Freiheit! Die Freiheit der Erwachsenen heißt Verantwortung “.9 Eindrücklich kommt diese lebensgeschichtliche Fokussierung in seiner Antrittsrede als Bundespräsident zum Vorschein, indem er Freiheit als „mein Lebensthema“ bezeichnet und auf sein Engagement im Rahmen der „friedlichen Freiheitsrevolution"10 verweist.

\footnotetext{
${ }^{5}$ Vgl. die ausgesprochen aufschlussreichen bildungstheoretischen und auch an konkreten Personen ausgerichteten Beiträge in Christoph Bizer u. a. (Hg.), Sehnsucht nach Orientierung. Vorbilder im Religionsunterricht, JRP 24. Neukirchen-Vluyn 2008 sowie Konstantin Lindner, In Kirchengeschichte verstrickt: Zur Bedeutung biografisch akzentuierter Zugänge für die Thematisierung kirchengeschichtlicher Inhalte im Religionsunterricht. Göttingen 2007.

${ }^{6}$ Peter Dausend, Wessen Präsident ist das? (28.11.2012), in: http://www.zeit.de/2012/49/Personalpolitik-Joachim-Gauck (Stand: 11.1.2013).

7 Joachim Gauck, Freiheit. Ein Plädoyer. München 2012, 3.

${ }^{8}$ A. a.0., 5.

${ }^{9} \mathrm{http}: / /$ www.uni-rostock.de/aktuelles/pressemeldungen/detailansicht-pressemeldung/news-artikel/ auf-vielfaeltige-weise-mit-der-universitaet-rostock/(Stand: 8. 11. 2012)

10 Joachim Gauck, Rede nach der Vereidigung zum Bundespräsidenten (23. 3. 2012). In: http://www.bundespraesident.de/SharedDocs/Reden/DE/Joachim-Gauck/Reden/2012/03/

120323-Vereidigung-des-Bundespraesidenten.html (Stand: 5. 12. 2012); vom „Wert der Demokratie“ als Lebensthema und von sich als „reisender Demokratielehrer“ spricht Gauck in seinen Erinnerungen: Winter im Sommer - Frühling im Herbst. Erinnerungen. München ${ }^{5} 2011$, 327; das Motto „Freiheit“ findet sich auch schon auf einem Bewerberplakat Gaucks zur Volkskammerwahl 1990, vgl. Gauck, Winter im Sommer Frühling im Herbst, a.a.0., 232.
} 


\section{Eine exemplarische protestantische Freiheitsgeschichte}

Der Werdegang Joachim Gaucks lässt sich als eine erstaunliche und an einschneidenden Ereignissen und dramatischen Wendemarken reichhaltige protestantische Freiheitsgeschichte lesen. Dies gilt nicht erst für die erste Kandidatur 2010 und dann für die überraschende Wahl des ostdeutschen Theologen in das Bundespräsidentenamt im Jahr 2012. Sondern dies ist auch schon für seine Aktivitäten im Pfarramt, weit vor der friedlichen Revolution des Jahres 1989 und während der ersten Jahre der deutschdeutschen Vereinigung festzuhalten. Insbesondere seine kirchliche Arbeit im letzten Jahrzehnt der DDR und dann die Jahre als Beauftragter der nach ihm benannten Behörde zur Umsetzung des Stasi-Unterlagen-Gesetzes haben dieser Freiheitsgeschichte in besonderer Weise den Charakter eines weit reichenden Befreiungsversuchs im Blick auf die DDR-Geschichte gegeben: Denn vermutlich hat die Beharrlichkeit des ostdeutschen Pastors - und als solcher ist Gauck im allgemeinen Bewusstsein nach wie vor präsent - im Blick auf den öffentlichen Zugang und Umgang mit den Stasi-Akten zumindest für viele der betroffenen Opfer den Charakter eines das Recht einklagenden und befreienden Umgangs mit der eigenen Geschichte erlangt. Insbesondere durch diese Tätigkeit ist Gauck, der Mitbegründer des Neuen Forums war und darüber hinaus für kurze Zeit auch der ersten freigewählten Volkskammer der DDR angehörte, damit auch der größeren bundesdeutschen Öffentlichkeit eventuell bekannt geworden.

Es soll nun in diesem Zusammenhang nicht im Einzelnen auf die biografischen Weg- und Wendemarken Gaucks eingegangen werden, noch zumal sich dies in unterschiedlich umfangreichen Versionen nachlesen lässt. ${ }^{11}$ Sondern interessant ist für den religionsdidaktischen Transfer, wie Gauck selbst in seinen Rückblicken und aktuellen Stellungnahmen die eigene biografische Freiheitsgeschichte entfaltet und mit seinen politischen Aktivitäten sowie der Deutung der Gegenwart im neuen Amt des Bundespräsidenten verbindet. Für diese Auslegung kann zum einen auf seine eigenen Erinnerungen und eine kleine systematische Abhandlung zur Freiheitsthematik, auf diverse Reden und Interviews aus jüngerer Zeit sowie schließlich auf diverse Biografien und Darstellungen von anderer Seite zurückgegriffen werden.

Die autobiografische Darstellung Gaucks, die vor seiner Wahl zum Bundespräsidenten endet, kann und soll wohl auch aus seiner eigenen Sicht als eine lange Darstellung eigener und familiärer Freiheits- und vor allem Unfreiheitserfahrungen gelesen werden. Bereits als Junge erlebt Gauck, wie der eigene Vater in einer Willküraktion der sowjetischen Besatzer praktisch über Nacht der Familie entzogen wird und erst viele Jahre später wieder nach Hause zurückkehrt. Diese Erfahrung sensibilisiert schon den jungen Gauck seinem eigenen Verständnis nach für das aufziehende Unrechtsregime sozialistischer Partei- und Staatsherrschaft in der DDR. Dabei notiert er früh und aufmerksam das sehr andere Demokratie- und Freiheitsverständnis des Westens und stellt

${ }_{11}$ Vgl. Norbert Robers, Joachim Gauck. Vom Pastor zum Präsidenten. Die Biografie. Leipzig ${ }^{2} 2012$; Dieter Bub, Begegnungen mit Joachim Gauck, Der Mensch. Sein Leben. Seine Überzeugungen. Halle (Saale) 2012. 
bereits für die frühe Zeit der DDR eine eklatante Verletzung der Menschenrechte sowie erheblichen Konformitätsdruck der Erziehung zur sozialistischen Persönlichkeit ${ }^{12}$ fest. Dem galt es dann im eigenen Pastorenamt von christlicher Seite aus in Bildungs-, Jugend- und Kirchentagsarbeit kritisch zu begegnen - und dies mit der Überzeugung: „Die Kirche machte die Menschen freier und für das System nicht oder weniger verführbar". ${ }^{13}$

Dass dabei von staatlicher Seite aus die öffentliche Rolle der Kirche einerseits systematisch in Frage gestellt wurde, andererseits aber eine der wenigen obrigkeitskritischen Instanzen bildete, zählt zu einer der frühen Erfahrungen Gaucks im Pfarramt. Und dass er dabei jederzeit mit staatlicher Observierung rechnen musste, war ihm ebenfalls von Beginn seiner pastoralen Tätigkeit an deutlich. In diesem Staat und dieser Gesellschaft durfte es dem ideologischen Selbstverständnis nach keine unpolitischen Räume und Orte und Zeiten geben - es sei denn in den Refugien der Wochenend-Freizeitgestaltung. So konnte hier auch die Kirche nicht anders als in einer durch die Partei und den Staat definierten oder bestimmten bzw. überwachten, kontrollierten und dokumentierten Öffentlichkeit existieren. Die Freiheit erfuhr somit bis in den Alltag hinein ihre permanenten Gefährdungen und Begrenzungen von staatlicher Seite aus.

Wie ein roter Faden zieht sich allerdings zugleich die Überzeugung Gaucks durch seine Erinnerungen, dass ein Verlassen des eigenen Landes, der Heimat für ihn keine sinnvolle Option darstellen konnte - besonders schmerzlich scheint diese Entscheidung gerade angesichts der Ausreise der eigenen Kinder in den Westen gewesen zu sein, weil dies ja mit einer unabsehbar dauerhaften familiären Trennung verbunden sein sollte.

Gleichwohl sollte dies für Gauck nicht heißen, sich mit den DDR-Verhältnissen einfach zu arrangieren oder gar die von manchen eingenommene programmatische Haltung einer „Kirche im Sozialismus“ mit zu vollziehen. In seiner interessanten Deutung dieser Formel als bloßer „Ortsbestimmung“" ${ }^{14}$ beschreibt er die christliche und kirchliche Aufgabe hier darin, sich in den bestehenden Verhältnissen kritisch mit diesen auseinander zu setzen, ohne aber dem Land den Rücken zu kehren. Die Ausrichtung an den westlichen demokratischen Werten stellte dabei gleichsam den permanenten, aber einstweilen unerreichbaren Horizont des eigenen Engagements dar.

In all dem entwickelte sich bei Gauck schon zu DDR-Zeiten ein prinzipiell wertebezogener und zugleich wertekonservativer Grundansatz, der ihn zugleich alle radikalen und befreiungstheologischen Strömungen, sei es im Osten oder im Westen, als fragwürdig ansehen ließ - und dies unter der etwas schillernd anmutenden Selbstbezeichnung als „linker, liberaler Konservativer“15: In den kritischen Blick geraten hier über-

12 Vgl. Gauck, Winter im Sommer, a. a. 0., 162.

13 A. a. O., 131.

14 A. a. 0., 146.

15 A. a. 0., 326. 
deutlich die Friedensaktivisten der 1980er Jahre und deren illusorische Bereitschaft der mentalen und militärischen Abrüstungsversuche des demokratischen Westens. ${ }^{16}$

So zieht sich durch seine Überlegungen immer wieder die kritische Darstellung gegenüber einer Haltung der „Freiheit von“ im Sinn der egoistischen Durchsetzung des eigenen Willens und erst recht eine deutliche Ablehnung aller linken Verklärung sozialistischer Freiheitsideale. Gegenüber der sehr realen biografischen Erfahrung des Verlusts und der permanenten Begrenzung eigener Freiheitsspielräume betont er immer wieder die Notwendigkeit wechselseitiger Verantwortung und echter Gemeinschaft - und dies unter der fundamentalanthropologischen Prämisse der „Lebensform der Bezogenheit“ und als „zentrale Menschenmöglichkeit“. ${ }^{17}$

Die Frage ist, ob und in welchem Sinn Gauck eigentlich auf theologische Argumente für diese grundsätzliche Selbstpositionierung zurückgegriffen hat. Grundsätzlich ist zu vermerken, dass explizite Bezüge innerhalb der genannten Texte nur sehr am Rande vorkommen. So verknüpft er seine Überlegungen nicht systematisch mit grundlegenden Charakteristika eines christlichen Freiheitsverständnisses. Selbst in seiner kleinen systematischen Abhandlung zur Freiheit ist in theologischem Sinn wenig zu finden. Interessant wäre es vermutlich, die Predigten Gaucks daraufhin einmal näher zu studieren, in denen sich vermutlich mehr auffinden lässt, wie es die wenigen Predigtauszüge innerhalb der Biografie bereits andeuten. ${ }^{18}$

Nur nebenbei sei bemerkt, dass die Ausführungen Gaucks zu seinem eigenen Theologiestudium bzw. zu prägenden Figuren oder systematisch-theologischen Referenzen erstaunlich dünn ausfallen. Eine gewisse Ausnahme bildet hier die Tatsache, dass Gauck sich schon zu DDR-Zeiten Bonhoeffers nicht-religiöse Rede von Gott zugutehält, „um in einer zunehmend säkularisierten Welt die großen Themen des Glaubens zur Sprache zu bringen oder der glaubenslosen Welt den Glauben plausibel zu machen“. ${ }^{19}$ Überhaupt scheint Gaucks theologisches Ethos vor allem auf grundlegenden Einsichten Bonhoeffers zu beruhen, die er aber offenbar eher in der konkreten Praxis der Jungen Gemeinde als durch sein theologisches Studium vermittelt bekam. ${ }^{20}$ Inwiefern hinter seiner spezifischen Deutung der Rede von der „Kirche im Sozialismus“ etwa eine Orientierung an Luthers Zwei-Regimenten-Lehre steht, wird wenigstens nicht explizit gemacht.

Zugleich verweist Gauck aber immer wieder auf seine unbedingte Orientierung an einem säkularen Verfassungs- und Rechtsverständnis von Freiheit und den entsprechenden verbindlichen rechtlichen Garantien. In diesem Zusammenhang unterscheidet er in charakteristischer Weise „zwei Gesichter von Freiheit“, die sich für ihn gerade in der deutschen Wendezeit manifestiert haben. Die eine Form, „Freiheit von etwas“, markiert er mit Begriffen wie Anarchie, Revolte, Bindungslosigkeit, Herrschaftsfreiheit,

\footnotetext{
${ }^{16}$ Vgl. Gauck, Freiheit, a. a. O., 45. Deutlich wendet er sich auch immer wieder gegen alle Ansprüche eines Gutmenschentums, vgl. etwa Gauck, Winter im Sommer, a.a. 0., 314.

${ }^{17}$ Gauck, Freiheit, a.a. O., 29.

${ }_{18}$ Vgl. Gauck, Winter im Sommer, a. a. O., 140f, 205.

19 A. a. 0., 147.

20 Vgl. a.a. 0., $111 \mathrm{f}$.
} 
Pubertät und - mit Schiller - mit der Fratze der ungezügelten, terrorartigen Freiheit. Die andere, „Freiheit für etwas“, identifiziert er mit europäischer Aufklärung, Demokratie und Bürgerrechten. ${ }^{21}$

Die Linie einer starken Betonung der Menschenrechte als universeller Prinzipien ${ }^{22}$ bei einer gleichzeitig erheblichen Zurückhaltung gegenüber expliziten theologischen Begründungen zeigt sich auch in den Reden als Bundespräsident, was angesichts der säkularen Fassung des Amtes ja tatsächlich so angemessen wie wenig verwunderlich ist.

Von dieser menschenrechtlichen Deutung aus finden sich dann tatsächlich auch verschiedentlich Anschlussstellen zur christlichen Tradition und Begründungslinie, so etwa im, wenn auch sehr allgemeinen, Verweis auf die ,jüdisch-christliche Dogmatik“23 oder im signifikanten Analogieschluss zwischen „ecclesia semper reformanda“ und „Freiheit und Demokratie: als eine societas semper reformanda“.24

An verschiedenen Stellen seiner Reden findet sich eine durchaus eindrückliche religiöse Semantik. So schliesst etwa die Antrittsrede mit einem „Gott und den Menschen sei Dank“25 für das geschichtliche Erbe des Landes ab und die erste Weihnachtsansprache setzt tatsächlich mit der Weihnachtsgeschichte ein - wobei interessanterweise sogleich eine pluralistische Offenheit signalisiert wird, wenn Gauck einsetzt: „Viele von uns lesen und hören in diesen Tagen die Weihnachtsgeschichte“ und dann fortfährt, von Botschaften zu sprechen, „die nicht nur religiöse, sondern alle Menschen ansprechen. ,Fürchtet Euch nicht!', und: ,Friede auf Erden!'“.26 Innerhalb der gleichen Rede kommt die Doppelpoligkeit von säkularer und religiöser Sprache erneut zum Tragen, wenn der Aspekt der „Zuwendung“ in folgender Weise ausdifferenziert wird: „In der Sprache der Politik heißt das: Solidarität, in der Sprache des Glaubens: Nächstenliebe“. Die Rede endet mit dem Verweis auf den „Stern aus der Weihnachtsgeschichte“, der - wiederum doppelpolig - auf die Geburt des Menschenkindes und auf die Mitmenschlichkeit hinführe. Offenkundig ist diese theologisch-offene Deutung mit dem Verständnis eines von Furcht und Angst befreienden Gottes verbunden, wobei diese biblische Freiheitsgewissheit eben sowohl politische wie existenzielle Bedeutsamkeit trägt und gerade deshalb auch säkular in hohem Maße von Gauck anschlussfähig gehalten wird.

Insofern kann für Gaucks Freiheitsverständnis gesagt werden, dass sich ein theologisches und ein verfassungsmäßig garantiertes Freiheitsverständnis keineswegs gegeneinander abschließen, sondern wechselseitig deutungsoffen sind. Die „emanzipatorische Bedeutung der christlichen Freiheit“27 einerseits und das modernde Verständnis

${ }^{21}$ Vgl. dazu Gauck, Freiheit, a. a. 0., 11-20.

22 Vgl. a.a. 0., 52.

23 A.a. $0 ., 51$.

${ }^{24}$ Gauck, Winter im Sommer, a.a. 0., 340.

25 Gauck, Rede nach der Vereidigung zum Bundespräsidenten (23. 3. 2012), a.a. O.

26 Joachim Gauck, Weihnachtsansprache 2012 (25. 12. 2012), in: http://www.bundespraesident.de/SharedDocs/Reden/DE/Joachim-Gauck/Reden/2012/12/121225-Weihnachtsansprache.html (Stand: 5. 1. 2013).

${ }^{27}$ Hans G. Ulrich, Art: Freiheit. In: Martin Honecker u.a. (Hg.), Evangelisches Soziallexikon. Stuttgart 2001, 507. 
von „Freiheit als das Zusammentreffen von Gabe, Aufgabe und Selbstbestimmung““28 werden unverkennbar zusammengedacht.

Von hier aus stellen sich nun die entsprechenden didaktischen Fragen: Welche Möglichkeiten für den Kompetenzerwerb hinsichtlich des eigenen produktiven Umgangs mit Religion können durch eine solche Auseinandersetzung entstehen? Erweckt ein solcher biografieorientierter Bildungsprozess bei Schülerinnen und Schülern selbst eine Sensibilität für den Zusammenhang zwischen christlicher Haltung und politischem Handeln? Und kann ihnen auf diese Weise etwas von der existenziellen Dimension des Freiheitsthemas selbst nahe gebracht werden? Kurz gefasst: Was lässt sich in religionsdidaktischer Hinsicht von Gaucks Darstellung und gleichsam seiner Verkörperung der Freiheitsthematik lernen?

\section{Konkretisierungen des Kompetenzerwerbs im Blick auf die Freiheitsthematik}

Zwar stellt in den Oberstufenlehrplänen für den schulischen Religionsunterricht das Thema „Kirche und Staat“ eine lange bewährte Einheit dar. Allerdings sind eigene Themenschwerpunkte zur Rolle der Christen und Kirchen in der DDR bisher nur in wenigen Rahmenlehrplänen der Bundesländer zu finden und auch noch kaum in die Überlegungen zu den kirchengeschichtlichen Themen des RU eingegangen. ${ }^{29}$ Auch eine dezidierte Behandlung des Freiheitsthemas ist, blickt man auf die Lehrpläne der Oberstufe, nicht vorgesehen. Insofern kann sich eine intensivere Beschäftigung mit der protestantischen Freiheitsgeschichte Gaucks als eine wichtige Erweiterung in den gegenwärtigen Bildungsplänen erweisen.

Transferiert man dies nun auf die Frage des Kompetenzerwerbs und geht man dafür vom Kompetenzmodell der EKD aus ${ }^{30}$, so ergeben sich eine Reihe möglicher Ansätze und Ausdifferenzierungen. Erinnert sei hier daran, dass gemäß dieses Modells ja unterschiedliche Gegenstandsbereiche von Religion in den Blick kommen sollen: Sowohl die Erwartungen und Bedürfnisse von Jugendlichen hinsichtlich ihres eigenen Glaubens, die christliche Überlieferung und Lehre sowie die je einschlägigen religiösen, kulturellen und gesellschaftlichen Zusammenhänge.

Nun wirft Gaucks biografische Freiheitsgeschichte tatsächlich die Frage der persönlichen Glaubenspraxis ebenso auf wie die Frage nach der tieferen Bedeutung des christlichen Freiheitsbegriffs und schließlich auch die Frage nach der politischen Bedeutung von Religion in den gegenwärtigen gesellschaftlichen Zusammenhängen.

\footnotetext{
${ }^{28}$ A.a. 0., 508.

29 Die neuere Überblicksdarstellung von Harmjan Dam, Kirchengeschichte im Religionsunterricht. Basiswissen und Bausteine für die Klassen 5-10. Göttingen 2010, endet beispielsweise klassisch mit Nationalsozialismus und Ökumene. Ein Überblick und Hinweise, die aber vor allem Desiderate hinsichtlich der Materialien für den Unterricht markieren, finden sich bei Manfred Pirner, Kirche im Sozialismus. In: Rainer Lachmann/ Herbert Gutschera/Jörg Tierfelder (Hg.), Kirchengeschichtliche Grundthemen. Historisch - systematisch didaktisch. Göttingen ${ }^{3} 2010,324-341$.

30 Vgl. Kirchenamt der EKD (Hg.), Kompetenzen und Standards für den Evangelischen Religionsunterricht in der Sekundarstufe I. Ein Orientierungsrahmen.
} 
Anhand dieser personalen Freiheitsgeschichte lassen sich die fünf prozessbezogenen Kompetenzen religiöser Bildung, also 1. Wahrnehmungs- und Darstellungsfähigkeit, 2. Deutungsfähigkeit, 3. Urteilsfähigkeit, 4. Dialogfähigkeit und 5. Gestaltungs- und Handlungsfähigkeit als Zielhorizont auf die Freiheitsthematik hin konkretisieren.

Dies beginnt im Blick auf die beiden erst genannten Punkte überhaupt einmal damit, sich das notwendige Faktenwissen über den spezifischen Charakter des DDR-Staates, die Rolle und Situation der Kirche sowie die Ereignisse der friedlichen Revolution anzueignen. Von dort können die persönlichen Hintergründe und Motive von Gaucks Sozialisation und Familiengeschichte im Zusammenhang seiner Unfreiheitserfahrungen, seines Freiheitsverständnisses sowie seiner kritischen Aufmerksamkeit für die jeweiligen politischen Verhältnisse erfasst und gedeutet werden. Insbesondere die frühen Erfahrungen als Jugendlicher von familiären Verlust-, Umbruchs- und Desorientierungssituationen können auch für heutige Schülerinnen und Schüler eindrücklichen Charakter gewinnen. Nebenbei bemerkt ist eine solche Annäherung an die realen Verhältnisse der jüngeren ostdeutschen Geschichte auch dazu geeignet, Jugendlichen insbesondere im Westen einen wesentlichen Aspekt der gemeinsamen deutschen Geschichte vor Augen zu führen. Von daher liefert schon diese historische Beschäftigung nicht nur einen wesentlichen Beitrag zur Erinnerungskultur im Klassenzimmer, sondern dient Jugendlichen auch zu einem besseren Verständnis der unterschiedlichen deutschen Entwicklungstraditionen.

Der Zusammenhang von Glaube und politischem, freiheitsliebendem Engagement und dies gerade angesichts von Gaucks eigener systematisch-theologischer Zurückhaltung - macht es sinnvoll, auch Traditionen und Elemente eines spezifisch biblischen Freiheitsverständnisses und deren theologische Interpretation zu erfassen und einzuordnen.

Hinsichtlich der Kompetenz eigener Deutungs- und Urteilsfähigkeit kann die Beschäftigung mit Gaucks Überlegungen und seiner Praxis dazu beitragen, sich ein eigenes ethisch reflektiertes Urteil über den Zusammenhang eines christlichen und eines säkularen Weltumgangs zu bilden. Hier liefert das bei Gauck nachvollziehbare, durchaus pragmatische Arrangieren mit den damaligen politischen Verhältnissen gute Diskussionsgrundlagen. Anhand der realen Enttäuschungserfahrungen Gaucks über den familiären Verlust und der offenen Entscheidungsfrage nach dem eigenen Bleiben oder Gehen kann die Freiheitsthematik in einer gleichsam existenziellen Weise gemeinsam mit den Jugendlichen diskutiert werden. Dies kann sich zugleich durch die Integration der von Gauck hier immer wieder stark verwandten Begriffssemantik der Verantwortung konkretisiert werden, die an die Lebenswelt und Lebenserfahrungen von Jugendlichen fraglos sehr gut anschlussfähig ist.

Zugleich lässt sich hier auch die Frage nach möglichen christlichen Begründungen eines bestimmten widerständig-oppositionellen Verhaltens gut aufwerfen. In diesem Zusammenhang können etwa die Aktivitäten als Kirchenmann in der DDR und seine protestierend-protestantischen Aktivitäten Gaucks im Vorfeld der Wende als Ausgangspunkte für eine solche Reflexion über die eigenen Kritik- und Widerstandspoten- 
ziale dienen. Es scheint jedenfalls an der Zeit, das religionsunterrichtliche Thema des Widerstands in seinem Begründungszusammenhang zum Glauben an das göttliche Befreiungsgeschehen nicht mehr alleine der Betrachtung des Lebenswerkes Dietrich Bonhoeffers zu überlassen.

Zugleich kann damit die Frage nach der öffentlichen Verantwortung von Kirche, Kirchengemeinde, Pfarramt und Kirchenmitglied bei schwierigen ethischen Sachverhalten oder innerhalb politischer Unrechtssysteme aufgeworfen werden. So sind die folgenden Frage- und Diskussionsimpulse denkbar: Soll Kirche nur in politischen Extremsituationen handeln? Was sind eigentlich unter den gegebenen aktuellen Umständen gegenwärtige Extremzustände? Welche Rolle soll der Religion im säkularen Staat und im öffentlichen Leben überhaupt zukommen?

Im Sinn einer öffentlichen Kirche als Bildungsinstitution ${ }^{31}$ kann hier jedenfalls an konkreten Beispielen über die Möglichkeiten einer Kirche als Gegenöffentlichkeit und Gegengemeinschaft gegen Unfreiheit und politische Indoktrination kritisch nachgedacht werden. Zu fragen ist im Sinn eines Kontexttransfers auch, ob Gaucks Bestimmung des Verhältnisses von Staat und Kirche im Zusammenhang einer „Kirche im Sozialismus" ein hilfreiches Paradigma für die Bestimmung der Kirche innerhalb der gegenwärtigen Zivilgesellschaft darstellt. Erörtert werden kann hier auch, ob es möglicherweise für die von Gauck dialektisch benannte Haltung „zwischen Verweigerung und Anpassung“32 während der DDR-Zeit aktuelle Vergleichspunkte geben kann. Zweifellos aber ist von der Erinnerung an die protestantische DDR-Kirche als „Vertreterin der politisch Unterprivilegierten“33 auch in demokratischen Gesellschaften zu lernen.

Im Sinn der Kompetenz individueller Dialog-, Gestaltungs- und Handlungsfähigkeit kann insbesondere die konkrete Versöhnungspolitik im Zusammenhang der Arbeit der Gauck-Behörde zum Ausgangspunkt für die Frage des eigenen Versöhnungshandelns im sehr viel kleineren alltagsbezogenen Rahmen werden. Von dort aus lässt sich in einem gestalterischen Sinn durchaus gemeinsam mit Jugendlichen sondieren, ob und wenn ja, welche Formen von kritisch-politischen Netzwerkbildungen innerhalb der gegenwärtigen pluralistischen und zivilgesellschaftlichen Verhältnisse denkbar, ja vielleicht sogar notwendig sind.

Schließlich eröffnen sich Möglichkeiten, mit Jugendlichen anhand aktueller Reden Gaucks im Amt des Bundespräsidenten nach dem Zusammenhang von christlicher Haltung und der politischen Funktion in einem weltanschaulich neutralen Staat zu fragen. Hier kann jugendliche Deutungs- und Urteilsfähigkeit hinsichtlich des Zusammenhangs von Religion und Politik nochmals in besonderer Weise zum Thema werden. Zudem deuten einige Reden Gaucks aus jüngster Zeit darauf hin, dass sich von dort aus auch die interreligiöse Dimension staatlich-politischen Handelns gut veranschauli-

31 Vgl. Thomas Schlag, Öffentliche Kirche. Grunddimensionen einer praktisch-theologischen Kirchentheorie. Zürich 2012, hier $77 \mathrm{f}$.

${ }^{32}$ Gauck, Winter im Sommer, a.a. 0., 144.

33 A.a. 0., 126. 
chen lässt. Gerade im Blick auf die Integration von religiösen und kulturellen Minderheiten wird schon jetzt Gesprächsstoff für den kompetenten Umgang mit interkulturellen und interreligiösen Konflikten im Klassenzimmer geliefert. ${ }^{34}$ Dass sich Gauck in anderem Interviewzusammenhang eindeutig für die Schaffung von Lehrstühlen für Islamwissenschaften sowie für die Ausbildung von Lehrkräften für islamischen Religionsunterricht ausspricht, erscheint von dort her ausgesprochen konsequent. ${ }^{35}$

Schließlich sind es immer wieder die prägnanten, auf die eigene Biografie bezogenen Zitate Gaucks, mit denen sich im Religionsunterricht die existenzielle Dimension des Politischen und die politische Dimension des Existentiellen zur Sprache bringen lassen, so wenn es etwa heißt: „Ängste - so habe ich es gelernt in einem langen Leben - vermindern unseren Mut wie unser Selbstvertrauen, und manchmal so entscheidend, dass wir beides ganz und gar verlieren können, bis wir gar Feigheit für Tugend halten und Flucht für eine legitime Haltung im politischen Raum“36 - es kann davon ausgegangen werden, dass sich gerade diese Gattung von Äußerungen Gaucks aufgrund ihrer persönlichen Eindrücklichkeit für Jugendliche als wesentlich zu erschließen vermag. Und gleiches dürfte auch gelten, wenn man etwa das aus der Erinnerung an die Zeit existenzieller Unfreiheit gewonnene Zitat Gaucks einspielt: „Die Freiheit war nicht dort, wo ich lebte. Die Freiheit war in meinen Sehnsüchten, in meinen Gedanken. Hier wurde sie stark “. ${ }^{37}$ Von dieser in sich hochkomplexen biografischen Positionierung Gaucks lässt sich gemeinsam mit den Jugendlichen die Vielfalt von Deutungen der Exoduserfahrung und -hoffnung erschließen.

Zugleich ist allerdings im Sinn kritischer Urteilsbildung auch Raum für die kritischen Rückfragen und damit für eine entsprechende Auseinandersetzung mit Gaucks Freiheitsgeschichte zu eröffnen. Kurz gesagt, muss der bildungsorientierte Umgang mit protestantischen Persönlichkeiten, wie hell ihr Licht auch strahlen mag, immer in einem prinzipiell kritischen Sinn erfolgen - dies gilt erst recht, wenn Gauck inzwischen sogar das Label des politischen Missionars ${ }^{38}$ angeheftet wird.

Zugleich wirft diese protestantische Freiheitsgeschichte aber auch verschiedene Fragenkomplexe auf, von denen einige andeutungsweise benannt werden sollen, insofern sie bestimmte Grenzen des hier erkennbaren Freiheitsverständnisses betreffen. Jedenfalls wird sich ein Kompetenzerwerb gerade auch auf diese Weise einstellen:

\footnotetext{
${ }^{34}$ Beispielhaft sei verwiesen auf die Reden zur Gedenkfeier „Lichtenhagen bewegt sich“ zum 20. Jahrestags der fremdenfeindlichen Angriffe auf das „Sonnenblumenhaus“ in Rostock am 26. 8. 2012, http://www.bundespraesident.de/SharedDocs/Reden/DE/Joachim-Gauck/Reden/2012/08/120826-Rostock.html

(Stand: 2. 12. 2012) und an ein dokumentiertes Gespräch mit den Schülerinnen und Schülern des Jüdischen Gymnasiums Moses Mendelssohn, Berlin am 9. November 2012 im Gedenken an die landesweiten antisemitischen Pogrome vom 9. November 1938, http://www.bundespraesident.de/SharedDocs/Berichte/DE/JoachimGauck/2012/11/121109-Ausstellung-7xJung.html (Stand 2. 12. 2012).

35 Vgl. „Meine Seele hat Narben“, Interview (31.5.2012), in: http://www.zeit.de/2012/23/Interview-Gauck (Stand: 5. 12. 2012).

${ }^{36}$ Gauck, Rede nach der Vereidigung zum Bundespräsidenten, a. a. 0.

37 Gauck. Freiheit, a. a. O., $20 \mathrm{f}$.

38 Vgl. Robers, Vom Pastor zum Präsidenten, a. a. 0., $193 \mathrm{ff}$.
} 
Tatsächlich sprechen gute Gründe für die von Gauck vorgenommene Differenzierung zwischen einer gleichsam theonomen und einer autonomen Freiheitsbegründung. Die Befreiungsbedürftigkeit im lutherischen Sinn stellt sich sachgemäß anders dar als etwa das Recht auf Entfaltungsfreiheit, das auch unabhängig von der eigenen individuellen Einsicht Bestand hat und zudem nicht notwendigerweise mit einem Auftrag zum Handeln am Nächsten verbunden sein muss. Dennoch ist zu fragen, ob nicht ein Theologe auch im biografischen Rückblick tatsächlich ausführlicher inhaltliche und systematische Auskunft über seine eigene Orientierung geben müsste. So ließe sich im Unterricht die Frage bearbeiten, ob ein bestimmtes Handeln als Christ schon als Plausibilisierung dafür ausreicht, christliches Handeln in seinem Begründungssinn zu erfassen. Jugendtheologisch gewendet ist hier also die Frage nach der Möglichkeit expliziter Begründungen für ein bestimmtes politisch relevantes Handeln aus christlicher Überzeugung heraus angesprochen.

Im Blick auf Gaucks Beschreibung des westlichen Freiheitsverständnisses ist zu fragen, ob hier nicht auch die Gefahr der Verklärung der faktischen Verhältnisse besteht und die gegenwärtigen, Unfreiheit erzeugenden politischen Umstände unterbelichtet sind. Auffallend ist im Übrigen, dass in den bisherigen präsidialen Äußerungen und Reden Kommentierungen der politisch-ökonomischen Gesamtsituation nur sehr am Rande erfolgen und überhaupt das eigene Amt bisher in eher zurückhaltender Weise mit programmatischen Zeitansagen verknüpft wird. ${ }^{39}$ Eine gewisse Idealisierung des Freiheitsbegriffs kann dort kritisiert werden, wo insbesondere der Zusammenhang mit der Gerechtigkeitsdimension nicht intensiver hergestellt wird - ein Aspekt, der insbesondere im zeitlichen Umfeld von Gaucks zweiter Kandidatur zum Bundespräsidenten thematisiert wurde. ${ }^{40}$ Entsprechend kann das wiederum der Antrittsrede entstammende folgende Zitat zum Ausgangspunkt der kritischen Reflexion werden: „Freiheit ist eine notwendige Bedingung von Gerechtigkeit. Denn was Gerechtigkeit - auch soziale Gerechtigkeit - bedeutet und was wir tun müssen, um ihr näherzukommen, lässt sich nicht paternalistisch anordnen, sondern nur in intensiver demokratischer Diskussion und Debatte klären. Umgekehrt ist das Bemühen um Gerechtigkeit unerlässlich für die Bewahrung der Freiheit". ${ }^{41}$

\section{Religionsdidaktische Folgerungen in allgemeiner Absicht}

Wird in religionsdidaktischer Hinsicht die Frage nach politisch relevanten Bildungszielen und Umsetzungsprozessen gestellt, so ist damit weder eine Engführung auf ethische Fragen noch gar eine ideologiekritische Ausrichtung intendiert. Gerade die Art und Weise Gaucks, das eigene Freiheitsverständnis in theologischer und in säkularer

\footnotetext{
39 Vgl. P. Wittrock, Wo ist Gauck? (28.11.2012), in: http://www.spiegel.de/politik/deutschland/joachimgauck-bundespraesident-hat-noch-keine-grossen-akzente-gesetzt-a-869311.html (Stand: 5. 12. 2012).

${ }^{40}$ Vgl. Albrecht Müller, Der falsche Präsident. Was Pfarrer Gauck noch lernen muss, damit wir glücklich mit ihm werden. Frankfurt/M. 2012; vgl. in polemischem Sinn das Traktat des ehemaligen Redakteurs des Neuen Deutschland Klaus Huhn, Die Gauck-Behörde. Der Inquisitor zieht ins Schloss. Berlin 2012.

${ }^{41}$ Gauck, Rede nach der Vereidigung zum Bundespräsidenten, a.a. 0.
} 
Sprache so unaufgeregt wie eindeutig durchzubuchstabieren, kann auch für eine überzeugungsstarke und auskunftsfähige Praxis der Kommunikation des Evangeliums gute Orientierungsmaßstäbe liefern.

Eine politisch relevante Religionsdidaktik zielt folglich darauf ab, Lehrpersonen zu einer stärkeren Integration des Politischen auch im Religionsunterricht zu motivieren, andererseits diese dafür zu sensibilisieren, die Unterscheidung zwischen Religion und Politik sowohl zu wahren als auch zugleich fruchtbar zu machen. Religiöse Bildungstheorie erinnert mit guten Gründen daran, dass menschliches Handeln auch innerhalb dieser Praxis zwar nicht aus Religion, aber mit Religion geschehen soll und kann. ${ }^{42}$

So lässt sich gerade anhand des doppelpolig bestimmten Freiheitsbegriffs die Einsicht verdeutlichen, dass alles menschliche Handeln auch im Bereich des Politischen einerseits mit Grenzen des Machbaren und prinzipieller Unverfügbarkeit, andererseits mit Möglichkeitsräumen individuellen Urteilens und Handelns zu tun hat. Insofern kann gerade die Verbindung einer realistisch-pragmatischen Sicht auf die politischen Gegebenheiten und theologischer Auskunftsfähigkeit eine hochproduktive Spannung und erhebliche gesellschaftskritische und gesellschaftsverändernde Potenziale erzeugen.

Von Gauck her findet sich selbst eine interessante gleichsam theologisch pointierte Deutung dieser Einsicht: Immer wieder moniert er, dass das Feld des Politischen „von vielen Menschen in Deutschland gleichgesetzt [wird] mit der Suche nach dem Vollkommenen - theologisch gesprochen, dem Schalom. Viele Menschen denken, dass die Politik Verachtung verdient, weil sie uns nicht mit dem Eigentlichen, dem Ideal, zusammenbringt ${ }^{4}{ }^{43}$ Religiöse Bildung hat tatsächlich zu vermitteln, dass es im Bereich des Politischen nicht um die Erzeugung und Befriedigung von Heilsbedürfnissen geht, sondern um ein „Streben nach dem Gestaltbaren“.44 Zur Sprache gebracht werden kann dabei die Vorstellung des zur Freiheit ermächtigenden Gottes, der dadurch zugleich die denkbar engste Beziehung mit dem Menschen eingegangen ist und immer wieder neu eingeht. Insofern ist an dieser Stelle tatsächlich eine prophetische und befreiungstheologische Pointierung so möglich wie sinnvoll: „There is no freedom of God without the politics of justice and compassion, and there is no politics of justice and compassion without a religion of the freedom of God". ${ }^{45}$

Religionsdidaktisch eröffnet ein solcher Zugang Strategien für den Umgang mit lebensweltlichen Schlüsselfragen und -problemen der heutigen Jugendgeneration. So ermöglicht und erleichtert die an den existenziellen Lebensfragen orientierte Thematisierung der Freiheitsdimension die Verkoppelung von alltäglicher Selbstwahrnehmung und theologischer Deutungskommunikation bei den Jugendlichen selbst. Insofern ist die von Gauck immer wieder betonte notwendige Erinnerung an die jüngere deutsche

\footnotetext{
$\overline{42}$ Vgl. dazu Bernhard Dressler, Religionsunterricht ist nicht Politikunterricht. Warum religiöse Bildung dennoch einen Beitrag zur politischen Bildung leistet, in: ZPT 64 (2012), v.a. $294 \mathrm{ff}$.

${ }^{43}$ Gauck, „Meine Seele hat Narben“, a. a. O.

${ }^{44}$ Ebd.

45 Walter Brüggemann, The Prophetic Imagination. Minneapolis ${ }^{2} 2001,9$.
} 
Geschichte - und hier sowohl die an die negativen wie die positiven Seiten - auch in religiöser Bildungspraxis zukünftig noch stärker zu entfalten. Gerade der Religionsunterricht hat hier die besondere Verantwortung und auch das Potenzial, die jüdischchristlichen und kirchlichen Traditionsbestände in ihrer aktuellen theologischen Bedeutsamkeit um der Gestaltung der Gegenwart willen immer wieder neu ins Gespräch zu bringen. Dies kann aber nicht mit dem Ziel erfolgen, lediglich die Materialien einer längst geschehenen Vergangenheit künstlich am Leben zu erhalten, sondern deren kritische Kraft etwa in Fragen politischer Macht, Herrschaft und Eliten unter der Verheißung irdischer Befreiung und Rettung zur Sprache zu bringen ${ }^{46}$ : „High theology. It is the gospel; God is for us". ${ }^{47}$

Die in diese Einsichten sich einübende Haltung und der angedeutete Erwerb vielfältiger politisch relevanter Kompetenzen sind natürlich nicht nur für das schulisch-formelle, sondern auch für das außerschulische informelle Lernen bedeutsam. Insofern kann die Beschäftigung mit dieser protestantischen Freiheits- und Befreiungsgeschichte auch für die kirchliche Bildungs- und Jugendarbeit eine aktualitätsbezogene Form des Dialogs mit Jugendlichen über die öffentliche Bedeutung von Religion und Glaube sein. ${ }^{48}$

Dem Sinn und Anspruch einer solchen individuellen freien Gewissensbildung entspricht allerdings am besten eine prinzipiell zurückhaltende und immer kritische Orientierung an einzelnen Personen, selbst wenn sie als protestantische Lichtgestalten erscheinen mögen. Gauck selbst hat durchaus kritisch alle Ansprüche an ihn als gleichsam politischen Erlöser früh notiert und kritisch kommentiert. ${ }^{49}$ Man sollte insofern selbst die eindrucksvolle Lebensgeschichte Gaucks eher als Ermutigung und Denkanstoß denn als eine kleine Heiligengeschichte im politischen Gewand betrachten. Ansonsten liefe man bis in konkrete Bildungsprozesse hinein Gefahr, der medialen Erregungskultur auf den unprotestantischen Leim der Anbetung von Menschen zu gehen.

Die Thematisierung und Rede von Freiheit im Kontext religiöser Bildung, finde diese nun im schulischen oder im kirchlichen Kontext statt, darf jedenfalls selbst niemals verklärenden, sondern muss verantwortlichen Sinn tragen, ganz im Sinn der Leitfrage des im besten Sinn befreiungstheologisch wirkenden Gauck: „Und du, wozu bist du imstande, wofür willst du dich einsetzen? Wie willst du Freiheit gestalten?“.50

\footnotetext{
46 Vgl. dazu die interessante Konkretion von Lukas Bormann, Befreiung und Rettung. Das Politische in der lukanischen Vorgeschichte (Lk 1-2), in: Eckhart Reinmuth (Hg.), Neues Testament und Politische Theorie, Interdisziplinäre Beiträge zur Zukunft des Politischen. Stuttgart 2011, 98-113.

47 Brüggemann, The Prophetic Imagination, a. a. 0., 16.

${ }^{48}$ Hingewiesen sei allerdings auch auf die noch sehr ungeklärten Fragen eines Kompetenzerwerbs durch politische Bildung, vgl. Horst Biedermann/Fritz Oser, Politische Mündigkeit durch schulische Partizipation? Zur Entmythologisierung des Wirksamkeitsglaubens von Partizipation. In: kursiv. Journal für die politische Bildung 1/2010, 28-44. Die hier angesprochenen Probleme, etwa der empirisch keineswegs klar aufweisbare Zusammenhang von Partizipation und Verantwortung, der Transfer partizipativen Erfahrungslernens in einen anderen Bereich oder überhaupt das Phänomen zufälligen Lernens müssen auch der Theoriebildung eines auf Freiheit hin orientierten Kompetenzerwerbs intensiv zu denken geben.

49 Vgl. „Der Präsident? Hoppla, das bin ja ich!“, Interview (11.11.2012), in: http://www.welt.de/politik/ deutschland/article110884564/Der-Praesident-Hoppla-das-bin-ja-ich.html (Stand: 5. 1. 2013)

${ }^{50}$ Gauck, Freiheit, a. a. 0., 22.
} 